\title{
Study of dyselectrolytemia in patients with multiple myeloma
}

\author{
Madanika $\mathbf{P}^{1,}$ Malathi $\mathbf{M}^{2}$, Ramlingareddy ${ }^{3, *}$ \\ ${ }^{\mathbf{1}}$ Assistant Professor, Dept. of Biochemistry, PES Institute of Medical Sciences and Research, Kuppam, Andhra Pradesh, \\ ${ }^{2}$ Professor and HOD, ${ }^{3}$ Assistant Professor, Dept. of Biochemistry, Father Muller medical College, Mangalore, Karnataka, India
}

*Corresponding Author:

Email: ramlingreddy2020@yahoo.co.in

Received: $8^{\text {th }}$ March, 2018

Accepted: $22^{\text {nd }}$ March, 2018

\begin{abstract}
Multiple myeloma (MM) is a malignant disease of plasma cells characterized by proliferation of a specific myelomatous clone of cells. It is second most common hematologic cancer. Renal impairment is observed in nearly half of the patients with multiple myeloma and $20 \%$ progress to renal failure due to precipitation of excess proteins in tubuli. Hyperproteinemia is associated with low plasma water levels causing unrealistically low electrolyte levels. Hyponatremia is one of the most commonly observed electrolyte disturbance. Evaluation of dyselectrolytemia in these patients and association with renal disease is useful in management and helps in monitoring prognosis. We conducted a cross sectional study on 31 newly confirmed cases of Multiple Myeloma diagnosed by serum protein electrophoresis, bone marrow aspiration and biopsy in Mangalore. Electrolyte disturbances observed include hypercalcemia (13\%), hyponatremia (42\%) and hypokalemia (6\%). Hypoalbuminemia was observed in $83 \%$ of cases. Renal impairment was seen in 58\% cases. Serum osmolarity was low despite increased proteins. Electrolyte disturbances are not uncommon in multiple myeloma. Hyponatremia can be asymptomatic and demands careful evaluation before institution of therapy. It is important to measure serum osmolarity and osmolar gap and carefully decide the management of electrolyte imbalance in every case of multiple myeloma. The coexistent hyperproteinemia, hyperglycemia, hypertriglyceridemia and renal disease should be evaluated. Hyponatremia was common in our study group although association with proteins or renal disease was not statistically significant.
\end{abstract}

Keywords: Dyselectrolytemia in Multiple myeloma, Pseudohyponatremia.

\section{Introduction}

Plasma cell dyscrasias include a heterogeneous group of diseases characterized by the expansion of the number of monoclonal bone marrow plasma cells that produce monoclonal immunoglobulins. ${ }^{1}$ Multiple myeloma is characterized by proliferation of a specific myelomatous clone of cells that accounts for almost $10 \%$ of all hematologic malignancies.Multiple myeloma can be distinguished from others by the presence of histological, serological and radiological features and includes bone marrow with clonal plasma cells or histological confirmation of a plasmacytoma; a monoclonal protein in the serum or urine (unless the patient has a nonsecretory myeloma [3\% of patients]); and end-organ damage evidenced by renal insufficiency, hypercalcemia, anemia, or lytic bone lesions. ${ }^{2,6}$ Many times myeloma may be diagnosed during evaluation of unexplained renal disease.

It is second most common among all haematological malignancies accounting for $15 \%$. The incidence of this disease increases with age It is $4 /$ lac in the general population to $30 / \mathrm{lac}$ in over 25 years population. The worldwide ASR for incidence is 1.4/1, 00,000 population per year. In the US ASR for incidence is higher at 5.8/1, 00,000 population, and in India it is $0.7 / 1,00,000$ population amounting to about 6,800 new cases a year. Worldwide the 5 year prevalence of the disease is $4.3 / 1,00,000$ population. In India it is $1.4 / 1,00,000$ population.The annual incidence of multiple myeloma is 4.3 per 100,000 people. ${ }^{3}$

The occurrence of new cases is largely dependent on gender, age, and ethnicity. It is more common among males than females. Electrolyte disturbances are known to occur in multiple myeloma. A review of 56000 patients suggests the association to be $0.03 \%$ and these Studies indicate different etiologies for such disturbances. ${ }^{2,4}$ Sodium, chloride and potassium are all affected. ${ }^{15}$ Hyponatremia is one of the commonly observed electrolyte disturbance with multiple myeloma. ${ }^{2,7-12,15}$ It may be true or pseudohyponatremia. Pseudohyponatremia is a clinical condition characterized by increased fraction of protein or lipid in plasma thereby resulting in an artificially low plasma sodium concentration less than $135 \mathrm{mmol} / \mathrm{L}$ due to the presence of increased plasma lipid and/or increased plasma protein concentration. However in true hyponatremia there is true decrease in serum sodium with decreased serum osmolarity. (Hypotonic hyponatremia)

Studies suggest the association of Multiple myeloma with dyselectrolytemia due to the many etiologies including the following,

Renal impairment is seen in 50\% patients with multiple myeloma and $20 \%$ progress to renal failure ${ }^{5,6}$ Due to precipitation of Monoclonal antibodies or Bence-Jones protein in the tubuli, impairment of kidney function occurs.. This may cause dyselectrolytemia. 
Hyperproteinemia due to increase in plasma solids causes electrolyte exclusion effect at the expense of plasma water level causes unrealistically low electrolyte levels. Isoelectric $\mathrm{pH}$ of immunoglobulins that of plasma proteins, hence net charge of plasma proteins may change causing new electrolyte balance. .,2,7 $^{2}$ Monoclonal antibodies that are hydrated more than others cause not only increased plasma viscosity but further electrolyte imbalance. ${ }^{8}$ Hypoalbuminemia can cause depletion of effective circulatory volume triggers secretion of $\mathrm{ADH}$, which can induce hyponatremia. ${ }^{12,13}$

Hyperglycemia causes hyperosmolarity (>285 mOsm) in the vascular space resulting in an extracellular shift of water secondary to high solute concentrations causing dilution of electrolytes to restore osmotic balance. This can also occur due to exogenous infusion of hypertonic solutions such as mannitol. ${ }^{9,10}$

Increased triglycerides affect all three electrolytes due to electrolyte exclusion effect showing artifactual decreases as a result of hyperlipidemia. Sodium and chloride decreases by $1 \mathrm{mmol} / \mathrm{L}$ and $\mathrm{K}+$ by $\sim 0.04$ $\mathrm{mmol} / \mathrm{L}$ for each $10-\mathrm{mmol} / \mathrm{L}$ increase in total lipid concentration. ${ }^{9}$

Other causes like Nutritional deficiency, Drug associated (Sulphonylureas, Thiazides) etc are also responsible for dyselectrolytemia.

Evaluation of the dyselectrolytemia in these patients and association with renal disease is useful in management and helps in monitoring prognosis.

\section{Materials and Methods}

We conducted a cross sectional study on 31 confirmed cases of multiple myeloma diagnosed by serum protein electrophoresis, bone marrow aspiration and biopsy on treatment during 2009-2012 from oncology unit, Father Muller Medical College, Mangalore. Our objectives were to study the electrolyte disturbances, and find out the factors causing the electrolyte imbalances in multiple myeloma patients. Serum sample was obtained from patients under aseptic precautions after obtaining institutional ethical clearance. The following tests were performed.

1. Serum electrolytes analysed by Ion selective electrode methods by direct and indirect methods using Ilyte electrolyte analyser and Cobas c311, Roche diagnostics fully automated analyser respectively.

2. Serum protein and Albumin were analysed by biuret method and BCG method respectively

3. Serum creatinine by Jaffe's kinetic method

4. Blood Glucose by Hexokinase method

5. Serum TG by Glycerol phosphate oxidase peroxidase method

6. Serum osmolarity was calculated by formula, Serum osmolarity $=\left(2 \times \mathrm{Na}^{+}\right)+($Glucose $/ 18)+$ (BUN/2.8)
Data was presented in the form of percentages. Results analysed using Epi Info 7 software. Chisquare test and Pearson's correlation was used for analysis.

\section{Results}

Our study group consisted 19 females and 12 males indicating a greater prevalence among females. Most cases $84 \%$ cases were in the age group of $56-65 y$ rs. It was observed that $13 \%$ cases had hypercalcemia though majority $(68 \%)$ in our study group had normal calcium levels. 15 out of 31 patients $(48 \%)$ had dyselectrolytemia. Most common being hyponatremia affecting $42 \%$ of our study group and 2 patients had hypokalemia (Graph 1). We evaluated the renal profile of these patients and observed that creatinine levels > $2 \mathrm{mg} / \mathrm{dl}$ was observed in $58 \%$ of cases. Hyponatremia was not associated significantly with renal impairment $(\mathrm{p}=0.6858)$. High protein was seen in $77 \%$ cases. Hypoalbuminemia was observed in $83 \%$ of cases. Hyperproteinemia due to increased globulins was observed. Hyperproteinemia was associated with hyponatremia in $37.5 \%$ cases $(p=0.35409$, not significant). Association was observed between hypoalbuminemia and hyponatremia but was not statistically significant $(\mathrm{p}=0.2777)$.

\section{Graph 1: Distribution of electrolyte disturbances}

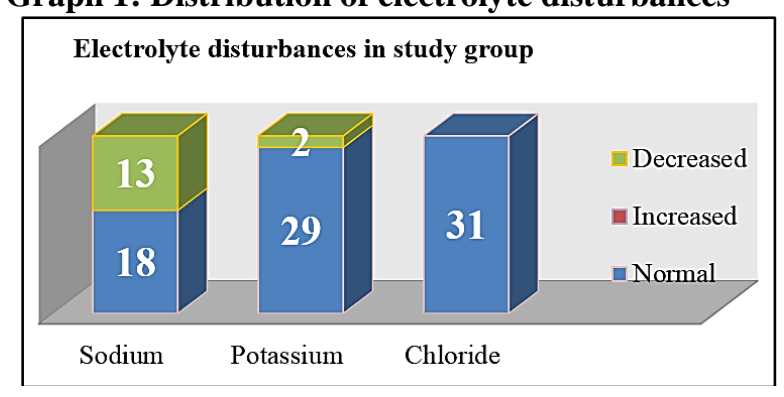

Low osmolarity was observed in these patients (55\%) despite increased proteins. Among those who had low osmolarity, most common electrolyte disturbance observed was hyponatremia with $65 \%$ patients and this association had strong positive correlation( $\mathrm{r}=0.8047)$. However most patients did not have any clinical manifestations of hyponatremia. It was suggestive of pseudohyponatremia rather than true hyponatremia. Hence we compared the serum sodium levels by both direct and indirect methods in patients with hyponatremia. ${ }^{13}$ A difference of $5-10 \mathrm{meq} / 1$ was observed. Even under normal circumstances by running controls a value of $2-5 \mathrm{meq} / \mathrm{L}$ difference is seen which is more predominant in hyponatremia due to Hyperproteinemia/ Hypertriglyceridemia, Hyperglycemia etc. In our study out of 31 cases, 24 had hyperproteinemia and distribution of all other abnormalities in biochemical parameters measured was as in Table 1. 
Table 1: Association of multiple myeloma with other possible causes of hyponatremia, * r=-0.0471 ** $r=-$ 0.2575

\begin{tabular}{|l|c|c|c|}
\hline S. No & Parameter & Levels & $\begin{array}{c}\text { Number } \\
(\% \text { out of Total sample size 31) }\end{array}$ \\
\hline 1 & $*$ Serum Total Protein & $>8 \mathrm{~g} / \mathrm{dl}$ & $24(77 \%)$ \\
\hline 2 & Serum Albumin & $<3.5 \mathrm{~g} / \mathrm{dl}$ & $26(84 \%)$ \\
\hline 3 & Plasma Random Glucose & $>140 \mathrm{mg} / \mathrm{dl}$ & $5(16 \%)$ \\
\hline 4 & Serum Triglycerides(Fasting) & $>150 \mathrm{mg} / \mathrm{dl}$ & $4(13 \%)$ \\
\hline 5 & $* *$ Serum Creatinine & $>2 \mathrm{mg} / \mathrm{dl}$ & $13(42 \%)$ \\
\hline
\end{tabular}

Among 13 cases that had hyponatremia, nine had hyperproteinemia (69\%) and none of these cases had hyperglycemia or hypertriglyceridemia. Serum creatinine was high in 6/13 patients (46\%). Low albumin was seen in 12 cases (92\%) as in Graph 2.

Graph 2: Association of hyponatremia with various factors in myeloma patients

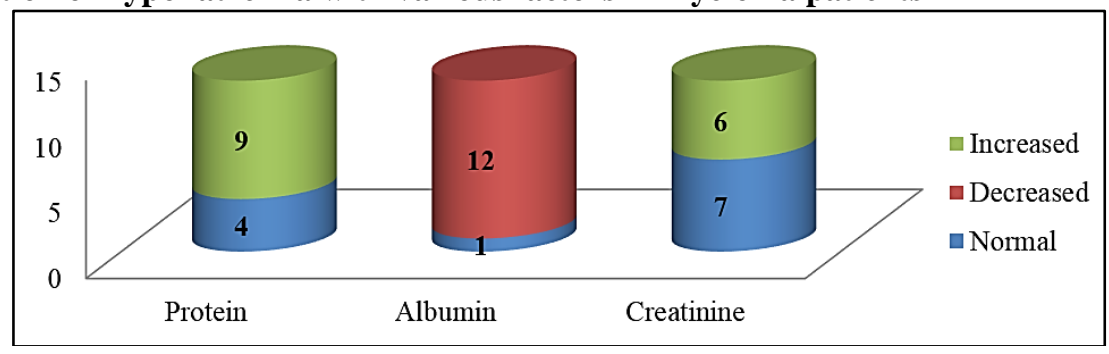

\section{Discussion}

Dyselectrolytemia in patients with multiple myeloma can be asymptomatic and demands careful evaluation before institution of therapy. Hyponatremia was seen in $42 \%$ patients as in other studies. ${ }^{2,4}$ Potassium and chloride were not significantly altered in our group. Hyponatremia in our group was associated with hypoalbuminemia, hyperproteinemia and also renal disease. Presence of normal/low serum sodium level in these patients should alert the possibility of hypernatremia and hypertonicity due to falsely low levels. It is important to measure electrolyte levels by both direct and indirect methods. In indirect ISE method sample is prediluted (1:10) and presented to the electrode as in modern high-throughput chemical analyzers because it requires very low sample volume, whereas direct ISE method undiluted sample (either whole blood, plasma or serum) is presented directly to the electrode as in blood gas analyzers, point of care in intensive care units, emergency rooms, operating recovery rooms, etc. Advantage is that the separation of plasma by centrifugation is not necessary and values are unaffected by the presence of abnormal amounts of lipid or protein in the sample..$^{15}$ It is important to know that the Blood plasma/serum is composed of $93 \%$ water and $7 \%$ non-aqueous solids most commonly being lipids and proteins. The\% water volume of plasma/serum is related to lipid and protein concentration by the following equation: \% water volume $=99.1-0.73$ protein $(\mathrm{g} / \mathrm{dL})-1.03$ lipid $(\mathrm{g} / \mathrm{dL})$

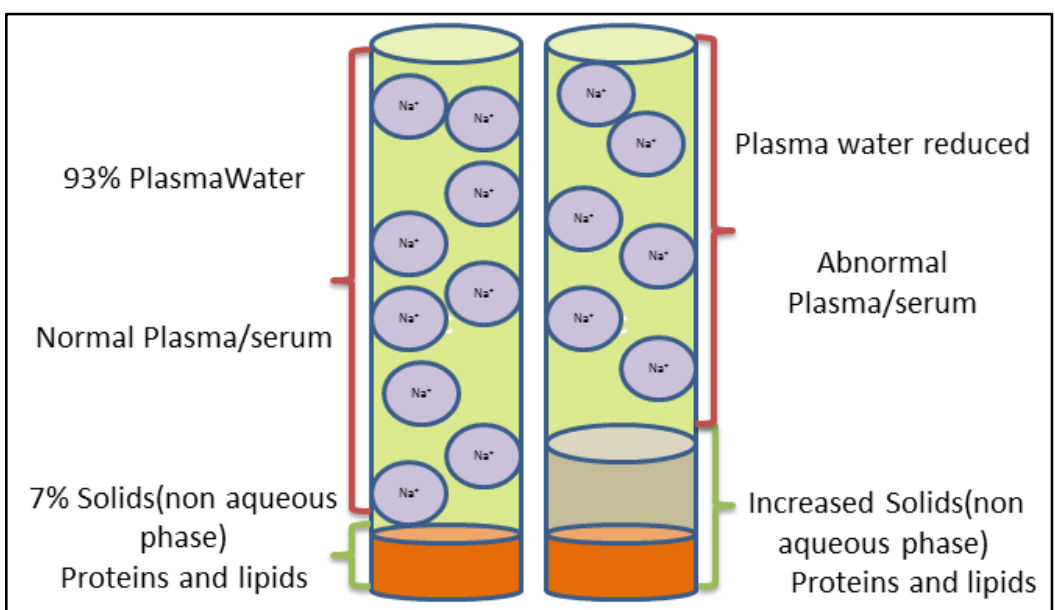

Fig. 1: Showing distribution of water and solids in plasma and electrolyte exclusion effect 
Sodium in serum or plasma mainly is present in liquid rather than solid phase. In health sodium concentration of plasma/serum water is $150 \mathrm{mmol} / \mathrm{L}$. considering when volume of solids is $7 \%$, the sodium concentration of total plasma/serum is $140 \mathrm{mmol} / \mathrm{L}$ $(150 \times 93 / 100)$. (Fig. 1) In direct ISE the volume of sodium plasma water is not altered and hence the increase in solids does not affect the sodium concentration. In indirect ISE due to dilution spurious sodium results may be obtained due to electrolyte exclusion effect as fewer sodium ions being presented to the electrode which is exaggerated in the presence of hyperproteinemia or hyperlipedemia. ${ }^{15}$ Patients with pseudohyponatremia are truly normonatremic and it is preferred to measure electrolytes by direct ISE rather than indirect method. In hyperglycemia hyponatrmia occurs due to osmotic effect. Both pseudohyponatremia and hypertonic hyponatremia should not be treated. Pseudohyponatremia can be observed in cases of multiple myeloma, waldenstorm's macroglobulinemia, IV administration of gamma globulins, hypergammaglobulinemia in liver disease, hypertriglyceridemia, and hypercholesterolemia and in the presence of certain abnormal lipoprotein X. ${ }^{15}$

The simplest way of confirming pseudohyponatremia is to reanalyze the sample using a direct ISE method as it was done in our study group; if value is normal, and excluded if it is similar to the original reduced indirect ISE result.

If direct ISE is not available, simultaneous calculation and measurement of plasma osmolarity is very useful. ${ }^{15}$ Measured osmolarity is normal in pseudohyponatremia but calculated osmolarity - based as it is on erroneously low plasma sodium result - is reduced. An increased osmolar gap suggests pseudohyponatremia. Though hyponatremia was common in our group its association with hyperproteinemia and other factors was not statistically significant. This may be due to small sample size in our group. However we could get a strong positive correlation between hyponatremia and decreased serum osmolarity (calculated) though there was hyperpoteinemia. Thus measured osmolarity and osmolar gap value also becomes important to obtain true value which was one of the limitation of our study.

\section{Conclusion}

Hyponatremia can be asymptomatic and demands careful evaluation before institution of therapy. In our study group $42 \%$ cases of multiple myeloma had hyponatremia. There was hyperproteinemia, hypoalbuminemia and renal disease associated. Presence of normal serum sodium level in these patients should alert the possibility of hypernatremia and hypertonicity. We measured the electrolytes by indirect and direct methods to rule out pseudohyponatremia. It is important to measure osmolarity and osmolar gap and carefully decide the management of electrolyte imbalance in multiple myeloma. The coexistent hyperproteinemia, hyperglycemia, hypertriglyceridemia and renal disease should be evaluated.

\section{References}

1. Stephen M, Korbet, Mewlin M, Shwartz. "Multiple Myeloma". J Am Soc Nephrol. 2006;17:2533-2545.

2. Miseta A, "Plasma electrolytes in multiple myeloma". Orv Hetil. 2001;142:1449-53.

3. Consensus document for management of multiple myeloma- prepared by ICMR subcommittee on multiple myeloma; 2017.Available from: http://icmr.gov.in/guide/cancer/MMICMR\%20Guidelines-24\%20Aug\%202015.pdf

4. Review: Could Multiple myeloma cause Blood electrolytes abnormal- from FDA reports; 2012. Available from: http://www.ehealthme.com/cs/multiple+myeloma/blood+ electrolytes+abnormal.

5. Joan Blade. Renal failure in Myeloma; 2000. Available from: http://myeloma.org/ArticlePage. action?articleId=34

6. Andia Banu-bradu A, Hortensia Ionita M, Ioana Ionita F, Corina Vernic, Claudiu Macarie. "Renal Disease in Multiple Myeloma", TMJ. 2011;61(1):32-36.

7. Dimeski G, Barnett RJ, "Effects of total plasma protein concentration on plasma sodium, potassium and chloride measurements by an indirect ion selective electrode measuring system”. Crit Care Resusc. 2005;7(1):12-15.

8. Gheun-Ho Kim. "Pseudohyponatremia: Does It Matter in Current Clinical Practice?" Electrolyte \& Blood Pressure. 2006;4(2):77-82.

9. E. Lars Penne, Md Stephan Thijssen, M, Jochen G. Raimann, Md, Nathan W. Levin, Md, Peter Kotanko. "Correction of Serum Sodium for Glucose Concentration in Hemodialysis Patients With Poor Glucose Control." Diabetes Care, 2010, vol. 33(7)2010;e-91.

10. George Liamis, Evangelos Liberopoulos, Fotios Barkas, Moses Elisaf "Diabetes mellitus and electrolyte disorders" World J Clin Cases. 2014; 2(10):488-496.

11. Goce Dimeski, Peter Mollee and Andrew Carter Effects of Hyperlipidemia on Plasma Sodium, Potassium, and Chloride Measurements by an Indirect Ion-Selective Electrode Measuring System; 2000. Available from http://www.clinchem.org /content/52/1/155.full

12. Story DA, Morimatsu H, Egi M, Bellomo R. "The effect of albumin concentration on plasma sodium and chloride measurements in critically ill patients". Anesth Analg. 2007;104(4):893-897.

13. Jack H. Ladenson, Fred S. Apple, James J. Aguanno, David D. Koch "Sodium Measurements in Multiple Myeloma: Two Techniques Compared"; Clin chem. 1982;28(12),2383-2386.

14. G. Dimeski, R. J. Barnett. "Effects of Total Plasma Protein Concentration on Plasma Sodium, Potassium and Chloride Measurements by an Indirect Ion Selective Electrode Measuring System"; Critical Care and Resuscitation. 2005;7:12-15.

15. Spurious sodium results (1) - pseudohyponatremia; March 2015. Available from:

https://acutecaretesting.org/en/articles/spurious-sodiumresults-1-pseudohyponatremia.

How to cite this article: Madanika $\mathrm{P}$, Malathi M, Ramlingareddy. Study of dyselectrolytemia in patients with multiple myeloma. Int J Clin Biochem Res. 2018;5(3):397-400. 